Classification

Physics Abstracts

$02.40-05.90-05.20$

\title{
Symmetry properties of the Bethe and Husimi lattices
}

\author{
J. A. de Miranda-Neto and Fernando Moraes \\ Departamento de Física, UFPE, 50739 Recife, PE, Brazil \\ (Received 10 July 1992, revised 18 September 1992, accepted 6 October 1992)
}

\begin{abstract}
The traditional picture of the Bethe and Husimi lattices as structures embedded in a space of infinite dimensionality permits no account of their metric and symmetry properties. In a previous work, we followed the indication of Mosseri and Sadoc that such lattices can be embedded in two-dimensional hyperbolic spaces and studied their metric properties. In this work we use such a representation to identify the symmetries of the generic $q$-coordinated Bethe lattice and of its associated Husimi lattice and construct a generating algorithm that, besides the precise coordinates of the vertices, provides also a way of labelling them hierarchically. The addition of symmetry and of a metric background space to these lattices enrich their potentiality for use as substrate for model systems, since it permits a more detailed analytical treatment of the models studied.
\end{abstract}

\section{Introduction.}

Many theoretical problems of statistical mechanics and solid state physics can be solved exactly on hierarchical structures [1] like the Bethe [2] and the Husimi [3] lattices. The Bethe lattice is usually viewed as an infinite ramified network, presenting a total absence of closed rings, a constant vertex connectivity and having all vertices equivalent. On the other hand, the Husimi lattice can be derived from a Bethe lattice by decorating its vertices and bonds; if each bond of the Bethe lattice is replaced by a single vertex and each vertex by a polygon, we obtain its associated Husimi lattice. Therefore, the Husimi lattice is built by putting together polygons attached to each other by their vertices, introducing closed rings (the polygons themselves) into the Bethe lattice. For the particular case where the polygons are triangles the Husimi lattice is referred to as a cactus [4].

By construction, the hierarchical lattices described above present serious crowding problems; e.g., the number of vertices grows exponentially with the distance while the available space grows only as a power law, the exponent being the dimension of the underlying space. For this reason, the Bethe and Husimi lattices are usually referred to as «pseudo-lattices of infinite dimension $»[5]$, since they cannot be embedded in any Euclidean space of finite dimension without serious distortion in their bond angles and lengths [6]. On the other hand, as pointed out by Mosseri and Sadoc [7] these structures if embedded in a two-dimensional space of constant negative curvature (the hyperbolic or Lobachevsky plane $\mathrm{H} 2$ ) are regular (Bethe) or quasi-regular (Husimi) lattices of fixed bond angles and lengths. This is not in contradiction 
with the infinite-dimensional representation since the volume in $\mathrm{H} 2$ grows exponentially with the distance, a feature that makes its metric properties effectively infinite-dimensional [8].

In a previous work [9] we studied the metric properties of a threefold coordinated Bethe lattice and of its associated Husimi lattice (cactus) in the Mosseri-Sadoc representation. There we showed that in addition to the commonly used topological distance it is possible to define a geodesic distance on such hierarchical structures. Coordinates were assigned to the vertices and expressions for the geodesic distances between given vertices and a vertex chosen as the origin were obtained for both structures. Use of these expressions was done to calculate the radial distribution functions for both cases. Additional structural information on the Bethe and the Husimi lattices appears from the study of their symmetries, the subject of this work.

Kléman and Donnadieu [10] made use of the symmetries of the Lobachevsky space to describe mechanisms of defect formation in hyperbolic crystals (including the threefold coordinated Bethe lattice), a very important device in decurving non-Euclidean models for frustrated systems in the Kléman-Sadoc $[11,12]$ scheme. Hyperbolic crystallography has also played an important role in the study of films and surfaces $[13,14]$, and hyperbolic symmetries have even been found in Euclidean crystals [15]. This relevance of non-Euclidean symmetries to physical problems motivated us to investigate further symmetry aspects of the Bethe and Husimi lattices. Hence, in this work we study the generating symmetries of these lattices; we find the matrices that describe such symmetries and construct a generating algorithm for both lattices that makes their hierarchical character explicit. The analysis of these symmetries would certainly not be an easy task in the infinite-dimensional approach to the hierarchical lattices. The knowledge of the metric and symmetry properties of these hierarchical lattices permits their use in the investigation of the structural details of amorphous systems [16], an aspect not yet explored in conventional applications of the Bethe and Husimi lattices [1].

In section 2 of this work we give a brief introduction to the isometries of the hyperbolic plane providing the mathematical tools for section 3 , where we find the matrices that describe rotational and parabolic symmetries for the generic $q$-coordinated Bethe lattice and its associated Husimi lattice. In terms of these matrices we write a generating algorithm that carries the natural hierarchy of such structures. The algorithm provides a simple and elegant computational tool which may be useful for the study of a variety of symmetry-related problems on the hierarchical lattices. Finally, in section 4, we present our conclusions and perspectives.

\section{The isometrics of the hyperbolic plane.}

The geometry and the symmetries of the hyperbolic plane have appeared in a variety of models in statistical mechanics and in other branches of theoretical physics. Many authors have used such tools to study a variety of problems such as separation of phase transition mechanisms and the analytic control of the infrared behaviour of QCD [8], quantum chaos [17], the effect of negative curvature in the QFT of bosons [18], structure of geometrically frustrated phases [12], films and surfaces [13], etc. On the other hand, the hyperbolic plane has been used as a background space for non-Euclidean versions of Ising [19] and $X Y$ models [20, 21], coherent states [22], solar cosmogony [23], etc. This diversity clearly shows the importance and the cross-disciplinary character of hyperbolic geometry as a theoretical tool. In this section we give a brief sketch of the geometry and symmetries of the two-dimensional hyperbolic space. For a more detailed account of this subject see references [24-26].

The hyperbolic or Lobachevsky plane $\mathrm{H} 2$ is a two-dimensional surface of constant negative curvature, which can not be globally embedded in a three-dimensional Euclidean space. However, the embedding is possible if the three-dimensional space is Minkowskian. There it 
appears as one sheet of a two-sheeted hyperboloid that can be conformally mapped onto the Poincaré disc, defined on the complex plane as follows :

$$
\mathbb{D}=\{z \in \mathbb{C}:|z|<1\} \text {. }
$$

Each point inside the disc represents a point of the hyperbolic plane; the boundary of the $\operatorname{disc} \Omega$, known as the Absolute, represents the points of $\mathrm{H} 2$ at infinity. The geodesics are the arcs of circle orthogonal to $\Omega$. In figure 1 we show examples of geodesics and the possible types of their mutual positions in $\mathbb{D}$.

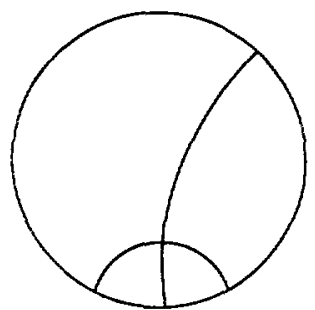

(a)

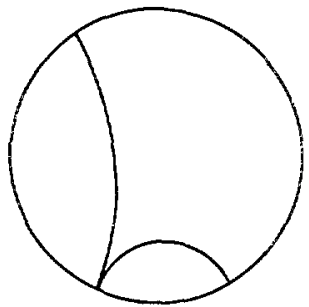

(b)

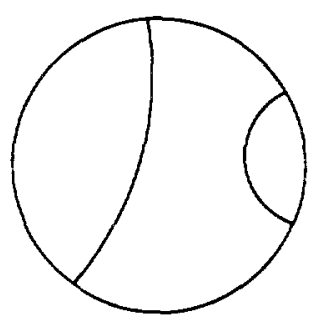

(c)

Fig. 1. - Types of mutual positions of geodesics in $\mathrm{H} 2$ in the Poincaré disc representation: a) intersecting, when the geodesics cross each other inside the disc ; b) parallel, the intersection point is on the Absolute, the boundary of the disc ; c) ultraparallel, the geodesics do not intersect inside or on the Absolute.

Like the Euclidean isometries the Lobachevskian ones can be described in terms of reflections about given geodesic axes. There are basically three types of isometries in $\mathrm{H} 2$ (see Fig. 2).

a) Elliptic isometry (or rotation) : double reflection about intersecting geodesics, equivalent to the motion on a circle centred at the intersection point (the sole fixed point of the operation).

b) Parabolic isometry : double reflection about parallel geodesics, equivalent to the motion on a curve called horocycle - a circle of infinite radius, centred at a point on $\Omega$, the fixed point of the operation. This transformation corresponds to a pure translation (in the Euclidean sense).

c) Hyperbolic isometry : double reflection about ultraparallel geodesics, equivalent to the motion on the common perpendicular to the reflection axes or on its equidistant curves (hypercycles). There are two fixed points lying on $\Omega$.

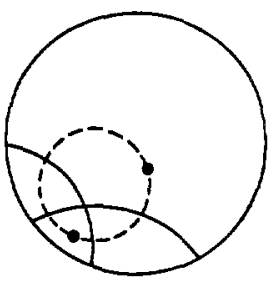

(a)

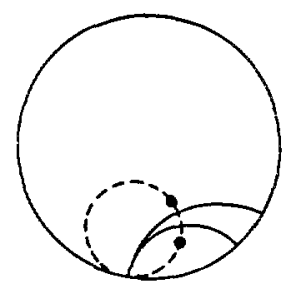

(b)

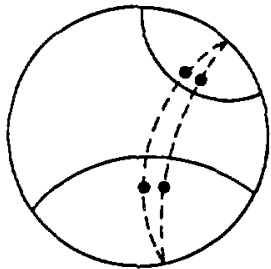

(c)

Fig. 2. - Types of isometries in $\mathrm{H} 2$ as represented by reflections in the Poincaré disc : a) elliptic ; b) parabolic ; c) hyperbolic. The equivalent motion of the reflected points takes place on the dashed circles. 
This geometric view of the isometries can be translated to an analytical form as follows. The isometries described above can be expressed as fractional linear transformations

where

$$
z \rightarrow z^{\prime}=\frac{\alpha z+\beta}{\beta^{*} z+\alpha^{*}},
$$

$$
|\alpha|^{2}-|\beta|^{2}=1 ; \quad \alpha, \beta \in \mathbb{C}
$$

They can also be expressed in a matrix form

$$
z \rightarrow z^{\prime}=\left[\begin{array}{cc}
\alpha & \beta \\
\beta^{*} & \alpha^{*}
\end{array}\right] z
$$

The nature of the transformation is determined by its fixed points which are equal to the ratio of the two components of each of the eigenvectors of the corresponding matrix. The identity matrices $\pm \llbracket$ shall not be considered because they represent the trivial mapping $z \rightarrow z$. This way the fixed points are the solutions of

$$
z=\frac{\alpha z+\beta}{\beta^{*} z+\alpha^{*}} \quad \text { or } \quad \beta^{*} z^{2}+\left(\alpha^{*}-\alpha\right) z-\beta=0
$$

The discriminant of this equation is easily seen to be $(\text { trace })^{2}-4$, where trace $=\alpha+\alpha^{*}$

The three basic types of isometries are thus :

a) elliptic isometry: if $\mid$ trace $\mid<2$ then there is one fixed point inside the disc. The other one, being outside, is not considered,

b) parabolic isometry: if $\mid$ trace $\mid=2$ then there is one double fixed point on $\Omega$,

c) hyperbolic isometry: if (trace) $>2$ then there are two distinct fixed points on $\Omega$.

The fixed points referred to above are very important to determine the matrices that describe intrinsic symmetries of the hierarchical lattices, the subject of the next section.

\section{The symmetries of the hierarchical lattices and the generating algorithm.}

In this section we identify the elliptic and parabolic symmetries of the $q$-coordinated Bethe lattice and of its associated Husimi lattice and build a generating algorithm that carries the natural hierarchy of such structures. For the sake of simplicity we start with the $q=3$ case and then generalise to arbitrary $q$. At the end of the section we relate this analysis to our earlier work [9] on the metric properties of hierarchical lattices.

Usually the Bethe and the Husimi lattices are viewed as infinite-dimensional structures, a characteristic that makes the idea of associating symmetry groups to them meaningless. The two-dimensional hyperbolic view presented in references [7, 9] allows a crystallographic analysis similar to what is done in ordinary Euclidean lattices. This approach makes possible to study the intrinsic symmetries of such hierarchical structures.

The Bethe lattice appears naturally in $\mathrm{H} 2$ as a limit case of its tiling by regular polygons [7]. These tilings (or tessellations) are denoted by the Schläfli [27] symbol $\{p, q\}$, meaning the structure obtained by placing $q$ regular polygons of $p$ edges around each vertex. In figure 3 we show the threefold coordinated lattices $(q=3)$ that can be build on a sphere $(p<6)$, on the Euclidean plane ( $p=6$, the hexagonal or honeycomb lattice) and on $\mathrm{H} 2$ as represented by the Poincaré disc $(p>6)$. The limit case $\{\infty, 3\}$ is easily identified with the well known threefold coordinated Bethe lattice. All polygons in a given hyperbolic $\{p, q\}$ are regular and of same size, in figure 3 they appear deformed due to an artifact of the Poincaré model. The model is conformal; that is, the angles presented are the actual angles in $\mathbf{H} 2$. In the $\{\infty, 3\}$ case all 
angles between any two adjacent edges of the tessellation are $2 \pi / 3$. The Bethe lattice when embedded in $\mathrm{H} 2$ has constant bond angles and lengths and preserves the connectivity and absence of rings of the infinite-dimensional version.

By definition, the centre of the Poincare disc coincides with the origin of the complex plane. Then, the most obvious symmetry of $\{\infty, 3\}$ is the $2 \pi / 3$ rotation about the origin

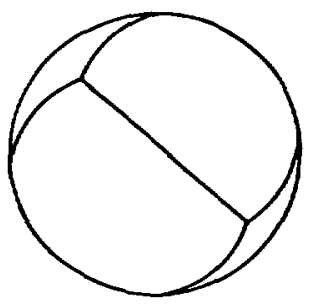

$\{3,3\}$

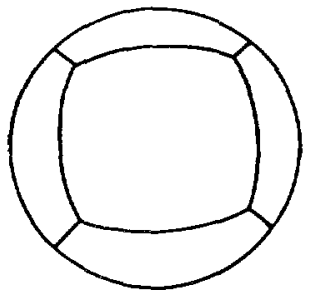

$\{4,3\}$

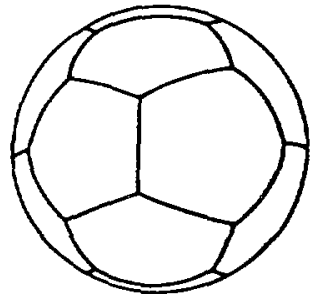

$\{5,3\}$
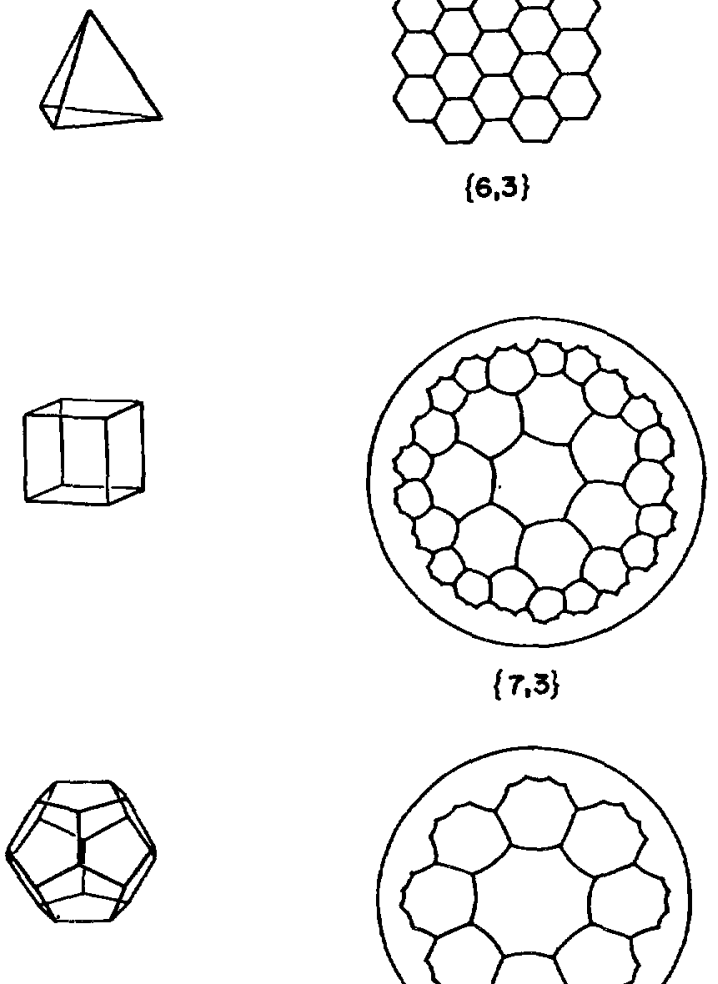

$\{7,3\}$

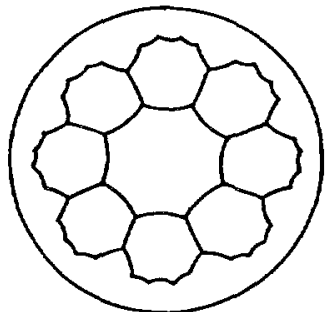

$\{8,3\}$
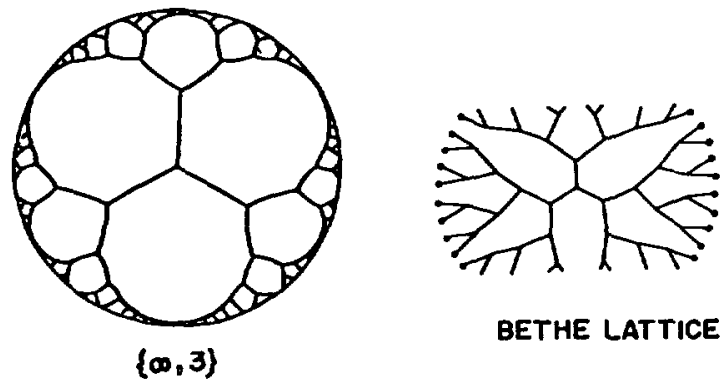

BeTHE latTice

Fig. 3. - Threefold coordinated lattices $\{p, 3\}$ defined on the sphere $(p<6)$, on the Euclidean plane $(p=6$, the hexagonal lattice) and on the hyperbolic plane $(p>6)$ as represented by the Poincare disc, and the Bethe lattice. 
$z_{0}$ which is the fixed point of the operation. That is, $S z_{0}=z_{0}$, where $S$ denotes the matrix that performs this rotation. This implies that the off-diagonal elements of $S$ vanish. The action of $S$ on a point $z \in \mathbb{C}$ is $\operatorname{such} z \rightarrow z \mathrm{e}^{i 2 \pi / 3} ;$ this and equation (2) gives

$$
S=\left[\begin{array}{cc}
\mathrm{e}^{\imath \pi / 3} & 0 \\
0 & \mathrm{e}^{-\imath \pi / 3}
\end{array}\right] .
$$

Notice that $\mid$ trace $S \mid=1<2$. Then the matrix $S$ represents an elliptic transformation, a rotation of $2 \pi / 3$ about the origin of the Poincare disc.

To find a parabolic symmetry we need some more elaboration. Notice that all the polygons that make up $\{\infty, 3\}$ are inscribed [28] in horocycles as seen in figure 4. In particular let us examine the horocycle containing the points ... $z_{-1}, z_{0}, z_{1}, \ldots, z_{\infty}$ (Fig. 4). The fixed point of the parabolic transformation $T$ corresponding to the motion on this particular horocycle is $z_{\infty}=\mathrm{e}^{i \pi / 3}$ which is the centre of the horocycle. That is, $T z_{\infty}=z_{\infty}$. Notice that $z_{\infty}$ is not the centre of the circle that represents the horocycle in the Poincare disc. As stated in section 2, since the horocycle is a circle of infinite radius, its centre is at infinity; i.e., on the boundary of the Poincare disc. On the other hand, since the polygon inscribed in the horocycle is regular, its centre coincides with the centre of the horocycle. This implies that the geodesic joining $z_{\infty}$ to the origin $z_{0}$ divides in half the internal angle $2 \pi / 3$.

In order to find $T$ we need another relation, say $T z_{0}=z_{1} ; z_{0}=0$ but we still need to find $z_{1}$. The geodesic distance $d$ between a point $z$ in $\mathbb{D}$ and the origin $z_{0}$ is given [29] by

$$
\cosh d=\frac{1+|z|^{2}}{1-|z|^{2}} .
$$

As on the surface of a sphere, each regular division $\{p, q\}$ of $\mathrm{H} 2$ requires a specific radius of curvature $R$. The relation between the edge length of a hyperbolic tessellation $\{p, q\}$ and its corresponding radius of curvature of $\mathrm{H} 2$ is [7]

$$
\cosh \frac{d}{2 R}=\frac{\cos \pi / p}{\sin \pi / q}
$$

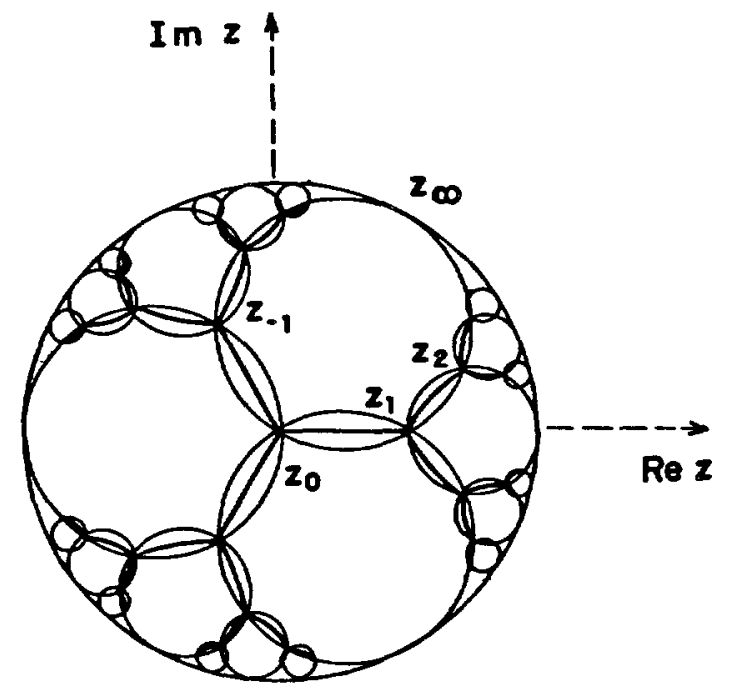

Fig. 4. - Chosen orientation of $\{\infty, 3\}$ with respect to the complex plane axes and some horocycles that circumscribe its infinite polygons (apeirogons). 
We choose our unit of distance such that $R=1$. For $p=\infty$ and using the identity $\cosh d=2 \cosh ^{2} \frac{d}{2}-1$, we obtain

$$
|z|=\cos \pi / q \text {, }
$$

where $q$ is an integer in the range $2<q<\infty$. For the tessellation $\{\infty, 3\}$ of figure 4 we find then $z_{1}=\cos \pi / 3=1 / 2$. Notice that for simplicity we choose the orientation of the axes such that $z_{1}$ lies on the real axis.

With the help of $T z_{\infty}=z_{\infty}$, where $z_{\infty}=\mathrm{e}^{i \pi / 3}, T z_{0}=z_{1}$ plus the condition given by equation (2) we find

$$
T=\frac{1}{\sqrt{3}}\left[\begin{array}{cc}
2 \mathrm{e}^{i \pi / 6} & \mathrm{e}^{-\imath \pi / 6} \\
\mathrm{e}^{i \pi / 6} & 2 \mathrm{e}^{-\imath \pi / 6}
\end{array}\right]
$$

Notice that $\mid$ trace $T \mid=2$ in agreement with the requirement of section 2 .

The vertices of $\{\infty, 3\}$ are generated by successive applications of the matrices $S$ and $T$ to a starting point in a hierarchical way as described below. If we take the starting point to be the origin $z_{0}$, it follows immediately that its first neighbours are $T z_{0}, S T z_{0}$ and $S^{2} T z_{0}$ as shown in figure 5a. If we now look at the first neighbours of $z_{1}=T z_{0}$, we find $T S^{2} z_{1}, T S z_{1}=$ $z_{0}$ and $T z_{1}$ (Fig. 5b). Proceeding further along the same branch, we find that the next generation of points is $T S^{2}\left(T S^{2} z_{1}\right), T S^{2}\left(T z_{1}\right), T\left(T S^{2} z_{1}\right), T\left(T z_{1}\right)$. That is, again $T S^{2}$ and $T$ applied to the previous generation. It follows that the branch growing out of $z_{0}$ through $z_{1}=T z_{0}$ and beyond is generated by the successive application of $T S^{2}$ and $T$ to all the points of a given generation as

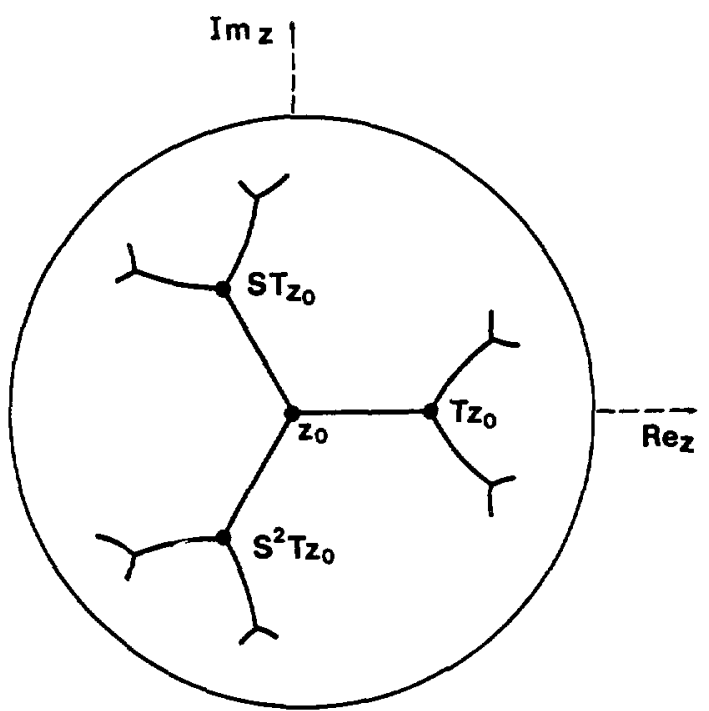

(a)

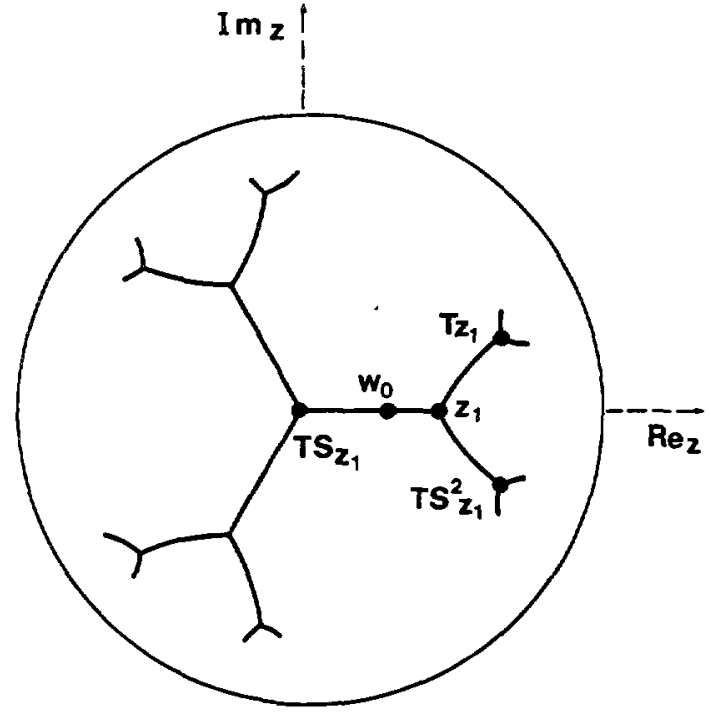

(b)

Fig. 5. - Finite portion of the threefold coordinated Bethe lattice $\{\infty, 3\}$ as represented in the Poincaré disc. a) The origin $z_{0}$ and its first neighbours in terms of the matrices $S$ and $T$; b) The first neighbours of the point $z_{1}$ in terms of the matrices $S$ and $T$, and the point $w_{0}$, the fixed point of TS. 
sketched below (notice that $T S^{2} z_{0}=T z_{0}=z_{1}$ )

$$
\begin{aligned}
& z_{0} \\
& z_{1} \\
& T S^{2} z_{1} \quad T z_{1} \\
& T S^{2}\left(T S^{2} z_{1}\right) \quad T S^{2}\left(T z_{1}\right) \quad T\left(T S^{2} z_{1}\right) \quad T\left(T z_{1}\right)
\end{aligned}
$$

and so on. Alternatively, the other branches out of $z_{0}$ can be generated by the pairs STS and $S T S^{2}$ or $S^{2} T$ and $S^{2} T S$. Or more simply, by rotations of the branch generated above under the action of $S$ and $S^{2}$ respectively.

The algorithm can be put in the diagrammatic compact form

$$
\begin{array}{ccc} 
& \mathrm{X}_{n} & \\
& & \\
T S^{2} \mathrm{X}_{n} & & T \mathrm{X}_{n}
\end{array}
$$

followed by iteration. $\mathrm{X}_{n}$ represents all the $2^{n-1}$ points of a given generation $n$. Notice that each new generation of points is obtained hierarchically by application of the operators $T S^{2}$ and $T$ to all points of the previous generation.

Thus, the matrices $S$ and $T$ suffice to generate $\{\infty, 3\}$; they actually form a discrete group with presentation [30] $\left\langle S, T ; S^{3},(T S)^{2}\right\rangle$ (notice that $S^{3}=(T S)^{2}=\mathbb{0}$ ), the so-called modular group [31]. A straightforward calculation gives $\mid$ trace $T S \mid=0<2$ implying that $T S$ is a rotation. Its fixed point is $w_{0}=2-\sqrt{3}$ which is the geodesic midpoint between $z_{0}$ and $z_{1}$. By inspection of figure $5 b$ it is evident that $T S$ denotes a rotation of $\pi$ around $w_{0}$ in agreement with Kléman [12]. The point $w_{0}$ has a fundamental role in the construction of Husimi lattices as will be described below.

Modelling of physical systems by or on Bethe lattices is not restricted to the threefold coordination case. Thus, it is important to extend the results found above to the generic coordination case, $\{\infty, q\}$. Here, invariance under $2 \pi / q$ rotations about $z_{0}$ yields

$$
S(q)=\left[\begin{array}{cc}
\mathrm{e}^{l \pi / q} & 0 \\
0 & \mathrm{e}^{-\imath \pi / q}
\end{array}\right]
$$

Analogously, we choose $T(q)$ to be the parabolic symmetry on the horocycle centred at $z_{\infty}=\mathrm{e}^{l \pi / q}$ Again $T(q) z_{\infty}=z_{\infty}$ and $T(q) z_{0}=z_{1}$ on the real axis, but now $z_{1}=\cos \pi / q$ from (6). These relations plus equation (2) results in

$$
T(q)=\frac{1}{\sin \pi / q}\left[\begin{array}{cc}
\mathrm{e}^{-\imath \theta} & \mathrm{e}^{i \theta} \cos \pi / q \\
\mathrm{e}^{-i \theta} \cos \pi / q & \mathrm{e}^{i \theta}
\end{array}\right],
$$

where $\theta=\left(\frac{2-q}{2 q}\right) \pi$. Notice that $|\operatorname{trace} S(q)|=2 \cos \pi / q<2$ and that $|\operatorname{trace} T(q)|=2$ in agreement with the specifications at the end of section 2 . 
The same reasoning that lead to algorithm (8) takes us to

$$
\begin{aligned}
& \mathrm{X}_{n} \\
& \begin{array}{llllll}
T(q) S^{2}(q) \mathrm{X}_{n} & T(q) S^{3}(q) \mathrm{X}_{n} & \cdots & \ldots & T(q) S^{q-1}(q) \mathrm{X}_{n} & T(q) \mathrm{X}_{n}
\end{array}
\end{aligned}
$$

for the generic $q$ case. Again all the $(q-1)^{n}$ points of each new generation are obtained from the $(q-1)^{n-1}$ points of the previous one, denoted by $\mathrm{X}_{n}$, by the application of the set of matrices $T(q) S^{2}(q), T(q) S^{3}(q), \ldots, T(q) S^{q-1}(q), T(q)$ in that order. As in the $q=3$ case, this is the generating algorithm for the branch growing out of $z_{0}$ towards $z_{1}$ on the real axis. The other $q-1$ branches can be obtained by successively applying $S(q)$ to this branch $q-1$ times. $S(q)$ and $T(q)$ generate a discrete group with presentation $\langle S(q), T(q)$; $\left.S^{q}(q), \quad(T(q) S(q))^{2}\right\rangle$. Here, $\mid$ trace $T(q) S(q) \mid=0<2$ plus $(T(q) S(q))^{2}=1$ imply that $T(q) S(q)$ is a rotation of $\pi$ around its fixed point. This algorithm besides the precise coordinates of the vertices on the disc also provides a way of labelling them hierarchically. This last feature is usually made by the so-called lexicographic ordering [32], frequently used in Hamiltonian models on the Bethe lattice, but without the geometric character given by our approach.

Now we turn to the symmetry properties of the Husimi lattice. This structure can be viewed as a limit case of the quasi-regular tiling $\left\{\begin{array}{l}q \\ r\end{array}\right\}$, when $r=\infty$. The general structure $\left\{\begin{array}{l}q \\ r\end{array}\right\}$ consists of regular polygons of two kinds (with number of edges $q$ and $r$ ), two of each at a vertex, arranged alternately. All the structures $\left\{\begin{array}{l}q \\ r\end{array}\right\}$ present a fixed coordination number equals to four. In figure 6, we show the quasi-regular structures $\left\{\begin{array}{l}3 \\ r\end{array}\right\}$ that can be built on a sphere $(r=4,5)$, on the Euclidean plane ( $r=6$, the Kagome lattice) and finally on the hyperbolic plane $(r>6)$ as represented by the Poincaré disc. The triangular Husimi cactus is obviously associated with the limit case $\left\{\begin{array}{c}3 \\ \infty\end{array}\right\}$. Notice that the generic Husimi lattice $\left\{\begin{array}{c}q \\ \infty\end{array}\right\}$ can also be obtained by joining the mid edges of the Bethe lattice $\{\infty, q\}$.

Since the vertices of the lattice $\left\{\begin{array}{c}q \\ \infty\end{array}\right\}$ are located at the middle of the edges of $\{\infty, q\}$, it follows that the finite polygons (we denote them by $\{q\}$ ) that make up the lattice are centred at the vertices of $\{\infty, q\}$. This implies the same rotational symmetry $S(q)$ for both $\left\{\begin{array}{c}q \\ \infty\end{array}\right\}$ and $\{\infty, q\}$ with the common fixed point $z_{0}=0$. On the other hand, the infinite polygons (the apeirogons $\{\infty\}$ ) of $\left\{\begin{array}{c}q \\ \infty\end{array}\right\}$ are circumscribed by horocycles centred at the same points the corresponding circumscribing horocycles of $\{\infty, q\}$ are. This implies the same fixed points $z_{\infty}$ and thus the same parabolic symmetry $T(q)$ for both $\left\{\begin{array}{c}q \\ \infty\end{array}\right\}$ and $\{\infty, q\}$. For the above reasons the generic algorithm we constructed for the case $\{\infty, q\}$ also generates $\left\{\begin{array}{c}q \\ \infty\end{array}\right\}$, with adequate choice of the starting point.

We would like to emphasise the importance of the starting point of the hierarchical algorithm. If we apply the hierarchical algorithm (8) starting from the point $z_{0}=0$, we obtain the threefold coordinated Bethe lattice. On the other hand, the corresponding Husimi cactus is obtained using the same algorithm (8), but beginning the iterative process from a distinct 


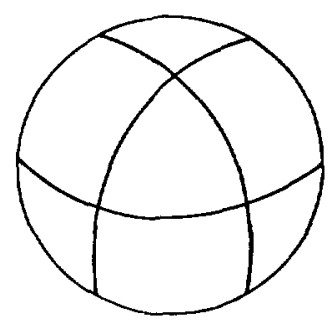

$\left\{\begin{array}{l}3 \\ 4\end{array}\right\}$

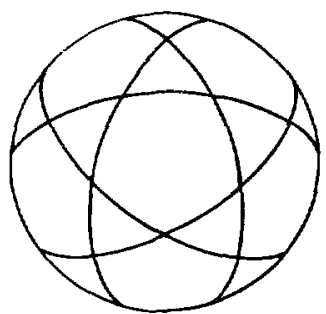

$\left\{\begin{array}{l}3 \\ 5\end{array}\right\}$

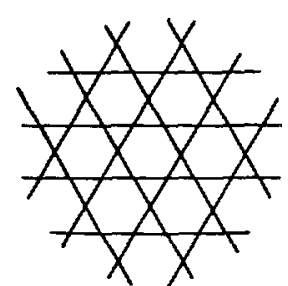

$\left\{\begin{array}{l}3 \\ 6\end{array}\right\}$-KAGOMÉ LATTICE

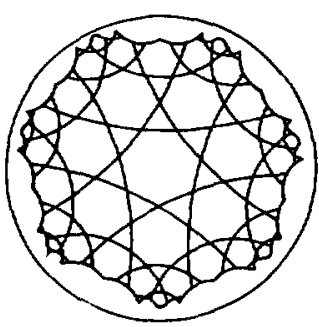

$\left\{\begin{array}{l}3 \\ 7\end{array}\right\}$

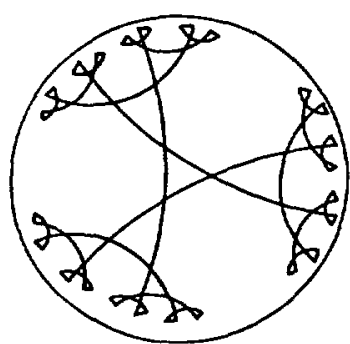

$\left\{\begin{array}{l}3 \\ 0\end{array}\right\}$

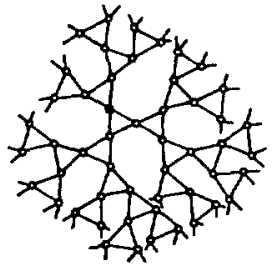

HUSIMI CACTUS

Fig. 6. - Quasi-regular structures $\left\{\begin{array}{l}3 \\ r\end{array}\right\}$ defined on the sphere $(r=4,5)$, on the Euclidean plane ( $r=6$, the Kagomé lattice) and on the hyperbolic plane $(r>6)$ as represented by the Poincaré disc, and the Husimi cactus.

starting point. By construction, the vertices of the cactus are located in between pairs of nearest neighbours of the Bethe lattice. Let $w_{0}$ be the vertex of the cactus located in the geodesic midpoint between the points $z_{0}$ and $z_{1}$, as shown in figure $5 \mathrm{~b}$ for the $q=3$ case. We keep the same notation for the generic $q$ case (i.e. $z_{0}$ is the point of $\{\infty, q\}$ at the centre of the disc, $z_{1}$ a first neighbour to $z_{0}$ and $w_{0}$ the midpoint) and once again choose the orientation of axes such that $z_{1}=T(q) z_{0}=\cos \pi / q$ lies on the real axis. As pointed out above for the $q=3$ case, the operation $T S$ is a rotation by $\pi$ around the midpoint between $z_{0}$ and $z_{1}$, that is around $w_{0}$. This is also true in the generic $q$ case and thus the starting point of the 
generic lattice $\left\{\begin{array}{c}q \\ \infty\end{array}\right\}$ is the fixed point of $T(q) S(q)$, which we find to be

$$
w_{0}=\frac{1-\sin \pi / q}{\cos \pi / q}
$$

For the triangular cactus $\left\{\begin{array}{c}3 \\ \infty\end{array}\right\}$ we find $w_{0}=\frac{1-\sin \pi / 3}{\cos \pi / 3}=2-\sqrt{3}$. Both structures $\left\{\begin{array}{c}3 \\ \infty\end{array}\right\}$ and $\{\infty, 3\}$ are generated by the algorithm (8), using the same $2 \times 2$ matrices $S$ and $T$, but with the starting points $z_{0}=0$ for the Bethe lattice and $w_{0}=2-\sqrt{3}$ for the Husimi cactus. Analogously, the generic Bethe lattice $\{\infty, q\}$ and its corresponding Husimi lattice $\left\{\begin{array}{c}q \\ \infty\end{array}\right\}$ are generated by the same matrices $S(q)$ and $T(q)$, arranged in the same generating algorithm. If we begin the iterative process applying the generating algorithm to the point $z_{0}=0$, we obtain the Bethe lattice ; on the other hand, if we do it with $w_{0}=\frac{1-\sin \pi / q}{\cos \pi / q}$ as the starting point, we obtain the Husimi lattice. Thus, the starting point assumes a very important role to distinguish the two hierarchical lattices. The reader may argue that being the hyperbolic plane a homogeneous space, all its points are equivalent thus changing the starting point of the algorithm would only displace the Bethe lattice and could not generate a different structure as we described above. However, when one chooses $S(q)$ and $T(q)$ as the generating symmetries one is implicitly breaking this homogeneity by the determination of their fixed points. This way, any point on $\mathrm{H} 2$ can be distinguished from any other point by reference to those fixed points; i.e., $\mathrm{H} 2$ acquires a reference frame. Notice that when we apply the operations $T(q)$ and $S(q)$ to the fixed point of $S(q), z_{0}$, we obtain $\{\infty, q\}$. If this is done to the fixed point of $T(q) S(q), w_{0}$, the lattice $\left\{\begin{array}{c}q \\ \infty\end{array}\right\}$ is obtained. Those are the fixed points of the generators of the group with presentation $\left\langle S(q), T(q) ; S(q)^{q},(T(q) S(q))^{2}\right\rangle$. The fixed point of $T(q), z_{\infty}$, under the same operations generates a cactus-like structure [28] of infinite coordination number, $\{q, \infty\}$.

Concluding this section we relate the symmetry properties found above to our previous work [9] where we studied metric aspects of hierarchical lattices using barycentric coordinates [27] on the projective plane. This coordinate system requires the use of a triangle of reference on whose vertices we put « masses » $t_{0}, t_{1}$ and $t_{2}$. Fixing the vertices of that triangle, any point of the projective plane can be described by a set $\left(t_{0}, t_{1}, t_{2}\right)$. Choosing an equilateral triangle of reference with the coordinates $(1,0,0),(0,1,0)$ and $(0,0,1)$ for its vertices, one obtains the Poincaré disc projection of $\mathrm{H} 2$. The Absolute is the circle, described by the equation $\Omega=x_{0} x_{1}+x_{1} x_{2}+x_{0} x_{2}=0$. Points satisfying $x_{0} x_{1}+x_{1} x_{2}+x_{0} x_{2}>0$ are inside the disc and hence represent points of $\mathrm{H} 2$.

In this coordinate system the vertices of the threefold coordinated Bethe lattice $\{\infty, 3\}$ and its associated Husimi cactus $\left\{\begin{array}{l}3 \\ \infty\end{array}\right\}$ are given by solutions of diophantine equations [9]. The vertices of $\{\infty, 3\}$ are given by the odd solutions of

$$
x_{0} x_{1}+x_{1} x_{2}+x_{0} x_{2}=3
$$

and the vertices of $\left\{\begin{array}{c}3 \\ \infty\end{array}\right\}$ are given by the integral solutions of

$$
x_{0} x_{1}+x_{1} x_{2}+x_{0} x_{2}=1
$$


The elliptic and parabolic symmetries described above for $\{\infty, 3\}$ and $\left\{\begin{array}{c}3 \\ \infty\end{array}\right\}$ are in this coordinate system given by the transformations

and

$$
\begin{gathered}
S:\left(x_{0}, x_{1}, x_{2}\right) \rightarrow\left(x_{1}, x_{2}, x_{0}\right) \\
T:\left(x_{0}, x_{1}, x_{2}\right) \rightarrow\left(2 x_{0}+x_{1},-x_{0}, x_{2}+2 x_{0}\right),
\end{gathered}
$$

which leave equations (12) and (13) invariant (as they should).

Expressing $\left(x_{0}, x_{1}, x_{2}\right)$ as a column vector, we find that $S$ and $T$ assume the matrix form

$$
S=\left[\begin{array}{lll}
0 & 1 & 0 \\
0 & 0 & 1 \\
1 & 0 & 0
\end{array}\right] \text { and } T=\left[\begin{array}{rrr}
2 & 1 & 0 \\
-1 & 0 & 0 \\
2 & 0 & 1
\end{array}\right]
$$

The generation of both $\{\infty, 3\}$ and $\left\{\begin{array}{l}3 \\ \infty\end{array}\right\}$ structures in this coordinate system is accomplished by the algorithm (8) using the $3 \times 3$ matrices $S$ and $T$. The starting points are now denoted by $z_{0}=(1,1,1)$ for the Bethe lattice and $w_{0}=(1,0,1)$ for the Husimi cactus [9]. The analysis of the intrinsic symmetries and the construction of a hierarchical algorithm for the Bethe lattice and the Husimi cactus also in barycentric coordinates complements the results obtained in our previous work.

\section{Concluding remarks.}

In this work we studied the intrinsic symmetries of the $q$-coordinated Bethe lattice and of its corresponding Husimi lattice which are hierarchical structures usually described as infinitedimensional. This analysis was made possible by replacing the infinite-dimensional Euclidean embedding space by a two-dimensional non-Euclidean space (the hyperbolic plane $\mathrm{H} 2$ ), which keeps infinite-dimensional characteristics while providing a metric background for those structures. There they become regular (Bethe) and quasi-regular (Husimi) curved crystals, with well defined symmetry groups. Based in rotational (elliptic) and translational (parabolic) intrinsic properties of the structures, we constructed an algorithm that provides the precise coordinates of the vertices and a practical way of labelling them hierarchically. The understanding of metric [9] and symmetry properties of hierarchical lattices, introduces the possibility of new uses for such structures, widening their already broad spectrum of applications in statistical mechanics and solid state physics.

Viewed as ideal hyperbolic crystals $\{\infty, q\}$ and $\left\{\begin{array}{c}q \\ \infty\end{array}\right\}$ may be used to model amorphous systems that share similarities with them like coordination, local order and dimensionality, along the lines proposed by Kléman and Sadoc [11]. For instance, the threefold coordinated Bethe lattice $\{\infty, 3\}$ may be related to $\mathrm{sp}^{2}$-bonded structures. Furthermore, such ideal curved crystals may be mapped from $\mathrm{H} 2$ onto flat Euclidean space, applying for example, the hierarchical method of inflation (or iterative flattening method-I.F.M.) as suggested by Sadoc and Mosseri [33]. On the other hand, more information on the physics of amorphous materials modelled with the help of $\{\infty, q\}$ and $\left\{\begin{array}{c}q \\ \infty\end{array}\right\}$, may be inferred from the study of electronic and acoustic properties of these hierarchical structures, through group theoretic arguments as done for three-dimensional spherical crystals [34-36].

As a concrete example, in a separate publication [16], we use this approach to hierarchical lattices to investigate structural details of boron trioxide $\left(\mathrm{B}_{2} \mathrm{O}_{3}\right)$ in its vitreous form. 
Experimental results [37] and theoretical models [38] indicate an overall two-dimensional character for this glass, which is composed by planar equilateral triangular units $\left(\mathrm{BO}_{3}\right.$ triangles) forming an open and low density structure. Decorating the vertices of $\{\infty, 3\}$ with boron atoms and putting oxygen atoms on its mid edges, corresponding to the vertices of $\left\{\begin{array}{c}3 \\ \infty\end{array}\right\}$, we build an ideal model for the glass along the lines of Kléman and Sadoc [11]. Using the symmetry and metric properties of the Bethe and Husimi lattices described in this paper and in reference [9], we make an analytical investigation of the ideal glass structure, obtaining good agreement with previous theoretical studies and experimental data.

Other interesting applications may be obtained by direct use of the generating algorithm. For example, it may be useful for a deeper investigation of physical systems where branching and bifurcation occur or in modelling growth processes. Besides hierarchical ordering those systems may present intrinsic non-Euclidean symmetries as for example phyllotaxis [39, 40], the quantum Hall effect [41] and Abelian lattice gauge theories with theta terms [42, 43]. In addition, one may use the symmetries of the generic Bethe and Husimi lattices to study the dimer and Ising problems on them, applying the Pffafian method [44], as done by Lund $e t$ al. [19] for a similar structure also embedded in $\mathrm{H} 2$. Finally, the knowledge of the symmetries of hierarchical structures suggest Fourier-Bloch-type of analysis, a powerful tool in conventional lattices but not yet explored in such structures.

\section{Acknowledgments.}

We are very much indebted to the referee for helpful comments and suggestions. We also thank $\mathrm{CNPq}$ and FINEP for financial support.

\section{References}

[1] ThORPE M. F., Excitations in Disordered Systems, NATO ASI series $\mathbf{B} 78$ (Plenum press, New York, 1982).

[2] Kurota M., Kikuchi R. and Watari T., J. Chem. Phys. 21 (1953) 434.

[3] Riddell R. J. and Uhlenbeck G. E., J. Chem. Phys. 21 (1953) 2056.

[4] Harary F. and Uhlenbeck G. E., Proc. Natl. Acad. Sci. U.S. 39 (1953) 315.

[5] Zallen R., The Physics of Amorphous Solids (John Wiley \& Sons, New York, 1983).

[6] Hughes B. D. and SAhimi M., J. Stat. Phys. 29 (1982) 781.

[7] Mosseri R. and Sadoc J. F., J. Phys. Lett. France 43 (1982) L249.

[8] Callan C. G. and Wilczek F., Nucl. Phys, B 340 (1990) 366.

[9] De Miranda-Neto J. A. and Moraes F., J. Phys. I France 2 (1992) 1657.

[10] Kléman M. and Donnadieu P., Philos. Mag. 52 (1985) 121.

[11] Kléman M. and Sadoc J. F., J. Phys. Lett. France 40 (1979) L569.

[12] Kléman M., Adv. Phys, 38 (1989) 605 and references therein.

[13] Sadoc J. F. and Charvolin J., J. Phys. Colloq. France 51 (1990) C7 319.

[14] Sadoc J. F. and Charvolin J., Acta Cryst. A 45 (1989) 10.

[15] JANNer A., Phys. Rev. Lett. 67 (1991) 2159.

[16] de Miranda-Neto J. A. and Moraes F., submitted to J. Phys. I France.

[17] Aurich R., Sieber M. and Steiner F., Phys. Rev. Lett. 61 (1988) 483 and references therein.

[18] Narayanan R. and Tracy C., Nucl. Phys. B 340 (1990) 568.

[19] Lund F., Rasseti M. and Regge T., Commun. Math. Phys. 51 (1976) 15.

[20] Dasmahapatra S., Mod. Phys. Lett. B 4 (1990) 1273.

[21] Link R., Mod. Phys. Lett. B 5 (1991) 1655.

[22] Perelomov A. M., Func. Analysts and its Applications 7 (1973) 215. 
[23] Rabinowitch A. S., Int. J. Theor. Phys. 30 (1991) 521.

[24] Coxeter H. S. M., Non-Euclidean Geometry (Univ. of Toronto Press, Toronto, 1947).

[25] BEARdon A. F., The Geometry of Discrete Groups (Springer Verlag, New York, 1981).

[26] Ford L. R., Automorphic Functions (Chelsea, New York, 1951).

[27] CoXeter H. S. M., Introduction to Geometry (John Wiley \& Sons, New York, 1961).

[28] Tóth L. F., Regular Figures (Pergamon, Oxford, 1964).

[29] Balazs N. L. and Voros A., Phys. Rep. 143 (1986) 109.

[30] The presentation of a discrete group is a notation for the set of its generators and the relations among them that produce the identity. See for example, Magnus W., Karrass A. and Solitar D., Combinatorial Group Theory: Presentations of Groups in terms of Generators and Relations (Dover, New York, 1976).

[31] Magnus W., Non-Euclidean Tesselations and Their Groups (Academic, New York, 1974).

[32] Peruggi F., J. Math. Phys. 25 (1984) 3303.

[33] SADOC J. F. and MOSSERI R., J. Phys. France 46 (1985) 1809.

[34] WidOM M., Phys. Rev. B 31 (1985) 6456.

[35] Mosseri R., Di Vicenzo D. P., Sadoc J. F. and Brodsky M. D., Phys. Rev. B 32 (1985) 3974.

[36] WIDOM M., Phys. Rev. B 34 (1986) 756.

[37] Johnson P. A. V., Wright A. C. and Sinclair R. N., J. Non-Cryst. Solids 50 (1982) 281 and references therein.

[38] Williams S. J. and Elliott S. R., Proc. R. Soc. Lond. A 380 (1982) 427 and references therein.

[39] Levitov L. S., Europhys. Lett. 14 (1991) 533.

[40] Rothen F, and Koch A. J., J. Phys. France 50 (1989) 1603.

[41] Lutken C. A. and Ross G. G., Phys. Rev. B 45 (1992) 11837.

[42] Cardy J. L., Nucl. Phys. B 205 (1982) 17.

[43] Shapere A. and Wilczek F., Nucl. Phys. B 320 (1989) 669.

[44] Kasteleyn P. W., J. Math. Phys. 4 (1963) 287. 